\title{
Aa. Vv., Peiresc et l'Italie, Actes du Colloque international
}

\section{Chiara Rolla}

\section{Q OpenEdition \\ 1 Journals}

\section{Edizione digitale}

URL: http://journals.openedition.org/studifrancesi/6605

DOI: 10.4000/studifrancesi.6605

ISSN: 2421-5856

\section{Editore}

Rosenberg \& Sellier

\section{Edizione cartacea}

Data di pubblicazione: 1 septembre 2010

Paginazione: 356-357

ISSN: 0039-2944

\section{Notizia bibliografica digitale}

Chiara Rolla, «Aa. Vv., Peiresc et I'Italie, Actes du Colloque international», Studi Francesi [Online], 161

(LIV | II) | 2010, online dal 30 novembre 2015, consultato il 13 janvier 2021. URL: http://

journals.openedition.org/studifrancesi/6605; DOI: https://doi.org/10.4000/studifrancesi.6605

Questo documento è stato generato automaticamente il 13 janvier 2021.

\section{(c) (i) (9)}

Studi Francesi è distribuita con Licenza Creative Commons Attribuzione - Non commerciale - Non opere derivate 4.0 Internazionale. 


\title{
Aa. Vv., Peiresc et l'Italie, Actes du Colloque international
}

\author{
Chiara Rolla
}

\section{NOTIZIA}

Peiresc et l'Italie, Actes du Colloque international (Naples 23-24 juin 2006), Paris, Alain Baudry, 2009, pp. 307.

1 Il volume contiene gli atti del Convegno internazionale che si è tenuto a Napoli nel 2006 e al quale ha presenziato Cecilia Rizza, stimata specialista delle relazioni italiane e degli interessi di Peiresc nel nostro Paese, nonché autrice di un saggio dedicato proprio ai rapporti tra Peiresc e l'Italia (Peiresc e l'Italia, Torino, Giappichelli, 1965). Questo personaggio, riscoperto solo sul finire del XIX secolo, contemporaneo almeno per una parte della sua vita di Montaigne con il quale presenta numerose affinità, grande erudito e partecipe della vita culturale dell'epoca, amico di Du Vair e di Gassendi, grazie agli studi qui presentati riemerge in tutta la sua complessità ed interesse (M. FUMAROLI, Introduction, pp. XI-XVII). Peiresc si è nutrito dell'enciclopedismo aristotelico dei grandi umanisti italiani: è stato a Padova dove ha conosciuto Gian Vincenzo Pinelli, frequentato la sua casa e soprattutto la sua biblioteca, analizzandone e studiandone i segreti della tecnica collezionistica (A. NUOvo, Ritratto di collezionista da giovane: Peiresc a casa Pinelli, pp. 1-17 e A. M. RAUGEI, Amor libri. Peiresc e la biblioteca di Gian Vincenzo Pinelli, pp. 19-29). A casa Pinelli conobbe anche Girolamo Aleandro il Giovane, considerato come il suo amico italiano più fedele: il loro carteggio, composto da più di trecento lettere, è, secondo Cecilia Rizza, fra le fonti più ricche per lo studio dei rapporti francoitaliani (S. DU CREST, Peiresc et Girolamo Aleandro il Giovane. Une étroite collaboration entre érudition et politique, pp. 61-74). Se paragonato con altri frequentatori della "République des Lettres" del suo calibro spicca subito la vastità dei contatti epistolari italiani di cui Peiresc poteva vantarsi (109, secondo Cecilia Rizza, contro i soli 12 annoverati dai fratelli Dupuy: J. DELATOUR, Les frères Dupuy et l'Italie, pp. 31-59). Insolitamente però 
Peiresc, all'apice della sua fama internazionale, non fu accolto dall'Accademia dei Lincei: E. SCHETTINI PIAZZA si interroga sulle ragioni del rifiuto di questa autorevole canditura e ne va alla ricerca delle possibili motivazioni (Una vocazione interrotta. Peiresc e i Lincei, pp. 75-90). Già il volume di Cecilia Rizza si era ampiamente soffermato sulle relazioni tra Peiresc e Galileo e ben si sa quanto il Nostro, sconvolto dalla notizia della condanna dello scienziato italiano, si sia a tal proposito espresso. Ciò nonostante S. GARCIA ritorna su questo argomento, alla luce soprattutto dell'apertura, nel 1998 in Vaticano, degli archivi della Congregazione per la dottrina della fede (Peiresc et l'affaire Galilée, ou le malaise d'un citoyen catholique de la République des lettres, pp. 91-104). Lo studio di Veronica CARPITA descrive la collezione di antichità etrusche, di monete e di incisioni raccolte dal magistrato e grande erudito italiano Natalizio Benedetti che Peiresc ebbe l'occasione di vedere durante il suo soggiorno in Italia del 1601; Carpita, inoltre, ricostruisce il metodo interdisciplinare elaborato da Peiresc per analizzare quei reperti e propone un'edizione critica della corrispondenza inedita tra i due eruditi (Natalizio Benedetti e Nicolas de Peiresc. Dal gusto per le "anticaglie" agli esordi dell'archeologia, pp. 105-156). Elena VAIANI ricostruisce le fasi principali degli studi condotti da Peiresc sulle piccole antichità greco-romane, la sua rete di contatti a Roma e i suoi scambi con il collezionista ed erudito Cassiano dal Pozzo e l'antiquario Claude Ménestrier (Nicolas Fabri de Peiresc, Claude Ménestrier e Cassiano dal Pozzo. Qualche esempio della fortuna delle piccole antichità tra Roma e Parigi, pp. 157-186). Peiresc fu anche un grande cultore della pittura e dell'architettura romane antiche, per le quali nutriva un interesse non solo estetico ma anche e soprattutto storico, come attestano i contibuti di H. LAVAGNE (Peiresc et la peinture romaine antique, pp. 187-204) e di F. LEMERLE (Nicolas-Claude Fabri de Peiresc et les ruines romaines, pp. 205-218). A. SCHNAPP, dopo aver brevemente illustrato lo stato della scienza antiquaria prima di Peiresc, presenta il metodo dell'antiquario scandinavo Ole Worm, che nel 1643 pubblica a Copenhagen il Danicorum Monumentorum libri sex, e ne mette in luce le affinità che esso condivide con quello di Peiresc: i due uomini incarnano, infatti, una stessa tipologia di erudito, desideroso di uscire dal proprio studio per lavorare sul campo e contestualizzare le proprie scoperte. ("La vertu des anciens Danois". Le modèle de Peiresc et l'influence italienne sur la formation de l'antiquarisme scandinave, pp. 219-241). L'articolo di I. DE CONIHOUT ripercorre e ripropone la straordinaria ricchezza della biblioteca di Peiresc e denuncia la mancanza a tutt'oggi di uno studio e di un'edizione degli inventari che la ricostruiscano in tutta la sua complessità (Du nouveau sur la bibliothèque de Peiresc, pp. 243-263). Lo studio di M. CERESA è un prezioso inventario della corrispondenza inedita tra Peiresc e alcuni cardinali bibliotecari presso la Biblioteca Apostolica Vaticana. Esso costituisce anche un'interessante analisi delle relazioni tra il Nostro e i prefetti di quella Biblioteca (Peiresc e la Biblioteca vaticana, pp. 265-277). Chiude il volume il contributo di P. N. MILLER nel quale viene messo in luce come il punto di vista del nostro erudito provenzale sulla cultura, la storia e la politica contemporanee fosse, a differenza di suoi illustri contemporanei del Nord, quali ad esempio Bacone o Cartesio, veramente ed intrinsecamente mediterraneo (From Anjou to Algiers. Peiresc and the Lost History of the French Mediterranean, pp. 279-291). 